\title{
Classical Zariski pairs
}

Article in Journal of Knot Theory and Its Ramifications · April 2012

DOI: $10.1142 /$ S0218216512500915

CITATION

1

1 author:

Aysegul Ozguner Ozturkalan

Abdullah Gül Üniversitesi

4 PUBLICATIONS 12 CITATIONS

SEE PROFILE
READS

87 


\title{
CLASSICAL ZARISKI PAIRS
}

\author{
AYŞEGÜL AKYOL \\ Department of Mathematics, Bilkent University, \\ 06533 Ankara, Turkey \\ ozguner@fen.bilkent.edu.tr \\ Received 7 June 2011 \\ Accepted 17 February 2012 \\ Published 12 April 2012
}

\begin{abstract}
We enumerate and classify up to equisingular deformation all irreducible plane sextics constituting the so called classical Zariski pairs. In most cases we obtain two deformation families, called abundant and non-abundant. Four sets of singularities are realized by abundant sextics only, and one exceptional set of singularities is realized by three families, one abundant and two complex conjugate non-abundant. This exceptional set of singularities has submaximal total Milnor number 18.
\end{abstract}

Keywords: Zariski pair; plane sextic; singularity.

Mathematics Subject Classification 2010: Primary 14H30; Secondary 14H45

\section{Introduction}

For the purpose of this paper, we define a Zariski pair as a pair of irreducible plane sextics $C_{1}$ and $C_{2}$ having the same set of singularities but not homeomorphic complements $\mathbb{P}^{2} \backslash C_{1}$ and $\mathbb{P}^{2} \backslash C_{2}$, see Definition 2.12 for details. Historically, the first example of such a pair was constructed by Zariski [16]. In this example, both curves have six cusps and one of them is of the so called torus type, i.e. its equation can be represented in the form $f_{2}^{3}+f_{3}^{2}=0$, where $f_{2}$ and $f_{3}$ are certain homogenous polynomials of degree 2 and 3, respectively. It is shown in [16] that the two curves differ by the fundamental groups $\pi_{1}\left(\mathbb{P}^{2} \backslash C_{i}\right), i=1,2$ and, moreover, by the Alexander polynomials $\Delta_{C}(t)$ (see Libgober [10] for the definition and basic properties of the Alexander polynomial of a plane algebraic curve). Following Degtyarev [2, 3], we call such pairs of sextics classical Zariski pairs.

Zariski's example was generalized in $[2,3]$, where it was shown that the Alexander polynomial of an irreducible plane sextic $C$ is $\Delta_{C}(t)=\left(t^{2}-t+1\right)^{d}, d \geq 0$. Moreover, the exponent $d$ is uniquely determined by the set of singularities of the 
curve unless this set of singularities is of the form

$$
\Sigma=e \mathbf{E}_{6} \oplus \bigoplus_{i=1}^{6} a_{i} \mathbf{A}_{3 i-1} \oplus n \mathbf{A}_{1}, \quad 2 e+\sum i a_{i}=6,
$$

in which case one may have $d=0$ or $d=1$. According to the value of $d$, such a sextic is said to be abundant (if $d=1$ ) or non-abundant $(d=0)$; this terminology is due to the fact that the value of $d$ is given by the superabundance of a certain linear system related to the curve. Thus, if two sextics $C_{1}, C_{2}$ form a classical Zariski pair, their common set of singularities must be as in (1.1).

According to $[2,3]$, just as in Zariski's original example, abundant sextics are necessarily of torus type, whereas non-abundant are not. In fact, it was recently shown in [5] that an irreducible plane sextic $C$ is of torus type if and only if its Alexander polynomial $\Delta_{C}(t)$ is non-trivial. This correspondence was first conjectured by Oka [8].

In this paper, we complete the classification of classical Zariski pairs and our principal result is the following theorem.

Theorem 1.1. The number of rigid isotopy classes of irreducible plane sextics realizing a set of singularities as in (1.1) is as follows:

- Each of the set of singularities $6 \mathbf{A}_{2} \oplus 4 \mathbf{A}_{1}, 2 \mathbf{A}_{2} \oplus 2 \mathbf{E}_{6} \oplus 2 \mathbf{A}_{1}, 4 \mathbf{A}_{2} \oplus \mathbf{E}_{6} \oplus 3 \mathbf{A}_{1}$, $3 \mathbf{E}_{6} \oplus \mathbf{A}_{1}$ is realized by only one rigid isotopy class which is abundant;

- The set of singularities $\Sigma=\mathbf{A}_{11} \oplus \mathbf{E}_{6} \oplus \mathbf{A}_{1}$ is realized by three distinct rigid isotopy classes, one abundant and two complex conjugate non-abundant;

- Any other set of singularities as in (1.1) is realized by exactly two rigid isotopy classes, one abundant and one not.

It is worth mentioning that the set of singularities $\Sigma=\mathbf{A}_{11} \oplus \mathbf{E}_{6} \oplus \mathbf{A}_{1}$ realized by three distinct rigid isotopy classes has submaximal total Milnor number 18 . (Recall that the total Milnor number of a simple plane sextic does not exceed 19.) To our knowledge, the corresponding non-abundant type is the first example of a non-maximizing configuration realized by two distinct rigid isotopy classes.

Theorem 1.1 is proved in Sec. 3. Section 2 contains the necessary preliminaries. First, following Nikulin's renowned paper [13], we recall some basic notions and facts concerning integral lattices and their discriminant forms. Then we describe the moduli spaces of plane sextics possessing a given set of singularities and, in particular, explain the construction of classical Zariski pairs; this part is mainly based on [4].

Remark 1.2. It would be interesting to have explicit equations for the curves having these sets of singularities, but I do not know how to write them down. In [8], Eyral and Oka give examples of equations for non-abundant types of seven sets of singularities as in (1.1) with $n=0$. In addition, in [14] and [6], other explicit constructions for some pairs of curves are found. 


\section{Preliminaries}

\subsection{Quadratic forms and integral lattices}

\subsubsection{Definitions and properties}

A finite quadratic form is a finite abelian group $\mathcal{L}$ with a map $q: \mathcal{L} \rightarrow \mathbb{Q} / 2 \mathbb{Z}$ satisfying $q(x+y)=q(x)+q(y)+2 b(x, y)$ and $q(n x)=n^{2} q(x), x, y \in \mathcal{L}, n \in \mathbb{Z}$, where $b: \mathcal{L} \otimes \mathcal{L} \rightarrow \mathbb{Q} / \mathbb{Z}$ is a symmetric bilinear form. Aut $\mathcal{L}$ is the group of automorphisms of $\mathcal{L}$ preserving $q$.

Any finite quadratic form can be decomposed into its primary components as orthogonal summands; $\mathcal{L}=\bigoplus \mathcal{L}_{p}=\bigoplus\left(\mathcal{L} \otimes \mathbb{Z}_{p}\right)$, summation over all primes $p$, where $\mathbb{Z}_{p}$ is the ring of $p$-adic integers. For any prime $p, \mathcal{L}_{p}$ is said to be the $p$-primary part of $\mathcal{L}$. Denote by $\ell(\mathcal{L})$ the minimal number of generators of $\mathcal{L}$.

Let $\left\langle\frac{m}{n}\right\rangle$ be the non-degenerate quadratic form on $\mathbb{Z} / n \mathbb{Z}$ sending the generator to $\frac{m}{n}$, where $\frac{m}{n} \in \mathbb{Q} / 2 \mathbb{Z}$ with $(m, n)=1$ and $m n=0 \bmod 2$. For an integer $k \geq 1$, let $\mathcal{U}_{2^{k}}$ and $\mathcal{V}_{2^{k}}$ be the quadratic forms on the group $\left(\mathbb{Z} / 2^{k} \mathbb{Z}\right)^{2}$ defined by the matrices

$$
\mathcal{U}_{2^{k}}=\left(\begin{array}{cc}
0 & \frac{1}{2^{k}} \\
\frac{1}{2^{k}} & 0
\end{array}\right) \quad \text { and } \quad \mathcal{V}_{2^{k}}=\left(\begin{array}{cc}
\frac{1}{2^{k-1}} & \frac{1}{2^{k}} \\
\frac{1}{2^{k}} & \frac{1}{2^{k-1}}
\end{array}\right)
$$

According to [13], each finite quadratic form is an orthogonal sum of cyclic summands $\left\langle\frac{m}{n}\right\rangle$ and copies of $\mathcal{U}_{2^{k}}, \mathcal{V}_{2^{k}}$.

A finite quadratic form is called even if $x^{2}$ is an integer for each element $x \in \mathcal{L}$ of order 2; otherwise it is called odd. A quadratic form is odd if and only if it contains $\left\langle \pm \frac{1}{2}\right\rangle$ as an orthogonal summand.

The Brown invariant of a finite quadratic form $\mathcal{L}$ is the residue $\operatorname{Br} \mathcal{L} \in \mathbb{Z} / 8 \mathbb{Z}$ defined by the Gauss sum

$$
\exp \left(\frac{1}{4} i \pi \operatorname{Br} \mathcal{L}\right)=|\mathcal{L}|^{\frac{-1}{2}} \sum_{i \in \mathcal{L}} \exp \left(i \pi x^{2}\right) .
$$

The Brown invariants of $p$-primary blocks are as follows:

$\operatorname{Br}\left\langle\frac{2 a}{p^{2 s-1}}\right\rangle=2\left(\frac{a}{p}\right)-\left(\frac{-1}{p}\right)-1, \operatorname{Br}\left\langle\frac{2 a}{p^{2 s}}\right\rangle=0($ for $s \geq 1$ and $(a, p)=1)$

$\operatorname{Br}\left\langle\frac{a}{2^{k}}\right\rangle=a+\frac{1}{2} k\left(a^{2}-1\right) \bmod 8($ for $k \geq 1, p=2$ and odd $a \in \mathbb{Z})$

$\operatorname{Br}_{2^{k}}=0 \operatorname{Br} \mathcal{V}_{2^{k}}=4 k \bmod 8$ (for all $k \geq 1$ ).

\subsubsection{Integral lattices}

An integral lattice is a free abelian group $L$ of finite rank with a symmetric bilinear form $b: L \otimes L \rightarrow \mathbb{Z}$. When $b(x, y)$ is understood, we use the multiplicative notation $x \cdot y$ instead of $b(x, y)$. A lattice $L$ is called even if $x^{2}=0 \bmod 2$ for all $x \in L$ or odd otherwise.

The transition matrix from one integral basis to another one has determinant \pm 1 , and hence the determinant of lattice $L$ can be $\operatorname{defined}$ as $\operatorname{det} L=\operatorname{det} b \in \mathbb{Z}$. 


\section{A. Akyol}

The lattice $L$ is non-degenerate if $\operatorname{det} L \neq 0$ and it is unimodular if $\operatorname{det} L= \pm 1$. The signature of a non-degenerate lattice $L$ is the pair $\left(\sigma_{+} L, \sigma_{-} L\right)$ of inertia indices of $L$. If $L$ is a non-degenerate integral lattice, the dual group $L^{*}=\operatorname{Hom}(L, \mathbb{Z})$ can be identified with the subgroup $\{x \in L \otimes \mathbb{Q} \mid x \cdot y \in \mathbb{Z}$ for all $y \in L\}$. The quotient group $L^{*} / L$ is called the discriminant group and is denoted by $\mathcal{L}$ or disk $L$. One has $|\mathcal{L}|=|\operatorname{det} L|$ and $\ell(\mathcal{L}) \leq \operatorname{rk}(L)$. The quotient group $L^{*} / L$ inherits from $L \otimes \mathbb{Q}$ a non-degenerate symmetric bilinear form $b: \mathcal{L} \otimes \mathcal{L} \rightarrow \mathbb{Q} / \mathbb{Z}$ and, if $L$ is even, its quadratic extension $q: \mathcal{L} \rightarrow \mathbb{Q} / 2 \mathbb{Z}$. Hence the discriminant group of an even lattice is a finite quadratic form.

Two non-degenerate integral lattices are said to have the same genus if their localization on $\mathbb{R}$-valued and $\mathbb{Q}_{p}$-valued forms are isomorphic.

Theorem 2.1 (see [13]). The genus of an even integral lattice $L$ is determined by its signature $\left(\sigma_{+} L, \sigma_{-} L\right)$ and discriminant form $\mathcal{L}$.

\subsubsection{Automorphisms of lattices}

Let us denote by $O(L)$, the group of auto-isometries of lattice $L$. Recall that $\sigma_{+} L$ is the dimension of any maximal positive definite subspace of vector space $L \otimes \mathbb{R}$. Any two maximal positive definite subspaces are oriented in a coherent way. The subgroup $O^{*}(L) \subset O(L)$ is the group of isometries preserving the orientation of maximal positive definite subspaces. Any isometry of $L$ preserves $\left(\in O^{*}(L)\right)$ or reverses $\left(\in O(L) \backslash O^{*}(L)\right)$ this orientation; in the latter case we call this isometry + -disorienting isometry.

Given a vector $a \in L$ with $a^{2} \neq 0$, the reflection is the automorphism $t_{a}: L \rightarrow L$ defined by $t_{a}: x \mapsto x-2 \frac{a \cdot x}{a^{2}} a$. The reflection $t_{a}$ is an involution, i.e. $t_{a}^{2}=\mathrm{id}$.

The image of the canonical homomorphism $O(L) \rightarrow \operatorname{Aut}_{\mathcal{L}}$ is denoted by $\operatorname{Aut}_{L} \mathcal{L}$.

\subsubsection{Existence and uniqueness of a lattice}

According to the following statement each $p$-primary finite quadratic form $\mathcal{L}_{p}$ is represented by a certain "minimal" $p$-adic lattice $K\left(\mathcal{L}_{p}\right)$.

Theorem 2.2 (see [13]). Let $\mathcal{L}_{p}$ be the p-primary part of $\mathcal{L}$. Then there exists a unique $p$-adic lattice $K\left(\mathcal{L}_{p}\right)$ of rank $\ell\left(\mathcal{L}_{p}\right)$ whose discriminant form is isomorphic to $\mathcal{L}_{p}$, except in the case when $p=2$ and $\mathcal{L}_{2}$ is even.

If $p=2$ and $\mathcal{L}_{2}$ is even, then there exist exactly two 2-adic lattices $\mathcal{L}_{2}^{1}$ and $\mathcal{L}_{2}^{2}$ of rank $\ell\left(\mathcal{L}_{2}\right)$ whose discriminant forms are $\mathcal{L}_{2}$.

Theorem 2.3 (see [13, Theorem 1.10.1]). Let $\mathcal{L}$ be a finite quadratic form and let $\sigma_{ \pm}$be a pair of integers. Then, the following four conditions are necessary and sufficient for the existence of an even integral lattice $L$ whose signature is $\left(\sigma_{+}, \sigma_{-}\right)$ and whose discriminant form is $\mathcal{L}$ :

(1) $\sigma_{ \pm} \geq 0$ and $\sigma_{+}+\sigma_{-} \geq \ell(\mathcal{L})$;

(2) $\sigma_{+}-\sigma_{-}=\operatorname{Br} \mathcal{L} \bmod 8$; 
(3) For each $p \neq 2$, either $\sigma_{+}+\sigma_{-}>\ell_{p}(\mathcal{L})$ or $\operatorname{det} K\left(\mathcal{L}_{p}\right) \equiv(-1)^{\sigma_{-}} \cdot|\mathcal{L}| \bmod \left(\mathbb{Z}_{p}^{*}\right)^{2}$;

(4) Either $\sigma_{+}+\sigma_{-}>\ell_{2}(\mathcal{L})$, or $\mathcal{L}_{2}$ is odd, or $\operatorname{det} K\left(\mathcal{L}_{2}\right) \equiv \pm 1|\mathcal{L}| \bmod \left(\mathbb{Z}_{2}^{*}\right)^{2}$.

The following theorem gives a partial answer to the problem of uniqueness of an even lattice in its genus.

Theorem 2.4 (see [13, Theorem 1.13.2]). Let $L$ be an indefinite even integral lattice, $\mathrm{rk} \geq 3$. The following two conditions are sufficient for $L$ to be unique in its genus:

(1) For each $p \neq 2$, either $\operatorname{rk} L \geq \ell_{p}(\mathcal{L})+2$ or $\mathcal{L}_{p}$ contains a subform isomorphic to $\left\langle\frac{a}{p^{k}}\right\rangle \oplus\left\langle\frac{b}{p^{k}}\right\rangle, k \geq 1$, as an orthogonal summand;

(2) Either $\operatorname{rk} L \geq \ell_{2}(\mathcal{L})+2$ or $\mathcal{L}_{2}$ contains a subform isomorphic to $\mathcal{U}_{2^{k}}, \mathcal{V}_{2^{k}}$, or $\left\langle\frac{a}{2^{k}}\right\rangle \oplus\left\langle\frac{b}{2^{k}}\right\rangle, k \geq 1$, as an orthogonal summand.

Theorem 2.5 (see [13, Theorem 1.14.2]). Let $L$ be an indefinite even integral lattice, $\operatorname{rk} L \geq 3$. The following two conditions are sufficient for $L$ to be unique in its genus and for the canonical homomorphism $O(L) \rightarrow \operatorname{Aut}(\mathcal{L})$ to be onto:

(1) for each $p \neq 2$, rk $L \geq \ell_{p}(\mathcal{L})+2$;

(2) either $\operatorname{rk} L \geq \ell_{2}(\mathcal{L})+2$ or $\mathcal{L}_{2}$ contains a subform isomorphic to $\mathcal{U}_{2^{k}}, \mathcal{V}_{2^{k}}$ as an orthogonal summand.

\subsubsection{Special lattices $\mathbf{U}$ and root systems}

The hyperbolic plane is the lattice $\mathbf{U}$ spanned by two vectors $u, v$ so that $u^{2}=v^{2}=$ $0, u \cdot v=1$. The hyperbolic plane is the only even unimodular lattice of signature $(1,1)$. Generalizing, we denote by $\mathbf{U}_{i}, i \in \mathbb{Z}^{+}$the lattice spanned by vectors $u, v$ with the property $u^{2}=v^{2}=0, u \cdot v=i$.

A Root system is a negative definite lattice generated by elements of square -2 . These elements are called roots. Every root system admits a unique decomposition into orthogonal sum of irreducible root systems. Irreducible root systems are $\mathbf{A}_{p}$, $p \geq 1, \mathbf{D}_{q}, q \geq 4, \mathbf{E}_{6}, \mathbf{E}_{7}, \mathbf{E}_{8}$, but we deal only with $\mathbf{A}_{p}, p \geq 1$ and $\mathbf{E}_{6}$. Their discriminant forms are disk $\mathbf{A}_{p}=\left\langle\frac{-p}{p+1}\right\rangle$, disk $\mathbf{E}_{6}=\left\langle\frac{2}{3}\right\rangle$.

\subsubsection{Extensions}

An even integral lattice $L$ containing even lattice $S$ for which $L / S$ is a finite abelian group, is called a finite index extension of $S$. For a finite index extension $L \supset S$, one has the inclusion $S \subset L \subset L^{*} \subset S^{*}$. The group $\mathcal{K}=L / S$ is an isotropic subgroup of $\mathcal{S}=S^{*} / S$ and $\mathcal{K}$ is called the kernel of the extension.

Two extensions $L_{1} \supset S$ and $L_{2} \supset S$ are isomorphic if there is an isomorphism $L_{1} \rightarrow L_{2}$ fixing S. For a fixed subgroup $A \subset O(S)$, A-isomorphism of extensions is an isomorphism whose restriction to $S$ belongs to $A$.

Proposition 2.6 (see [13]). Let $S$ be a non-degenerate even lattice, and fix a subgroup $A \subset O(S)$. The map $L \mapsto \mathcal{K}=L / S \subset \mathcal{S}$ establishes a one-to-one 


\section{A. Akyol}

correspondence between the set of A-isomorphism classes of finite index extensions $L \supset S$ and the set of $A$-orbits of isotropic subgroups $\mathcal{K} \subset \mathcal{S}$. Under this correspondence one has $\mathcal{L}=\mathcal{K}^{\perp} / \mathcal{K}$.

An isometry $f: S \rightarrow S$ extends to a finite index extension $L \supset S$ defined by an isotropic subgroup $\mathcal{K} \subset \mathcal{S}$ if and only if the automorphism $\mathcal{S} \rightarrow \mathcal{S}$ induced by $f$ preserves $\mathcal{K}$.

Another extreme case is when $S$ is a primitive non-degenerate sublattice of a unimodular lattice $L$. In this case $S^{\perp}$ is also primitive in $L$ and $L$ is a finite index extension of $S \oplus S^{\perp}$. Since disk $L=0$, the kernel $\mathcal{K} \subset \mathcal{S} \oplus \operatorname{disk} S^{\perp}$ is the graph of an anti-isometry $\kappa: \mathcal{S} \rightarrow \operatorname{disk} S^{\perp}$. Conversely, given a lattice $N$ and an anti-isometry $\kappa: \mathcal{S} \rightarrow \mathcal{N}$, the graph of $\kappa$ is an isotropic subgroup $\mathcal{K} \subset \mathcal{S} \oplus \mathcal{N}$ and the resulting extension $L \supset S \oplus N \supset S$ is a unimodular primitive extension of $S$ with $S^{\perp}=N$.

Let $s: S \rightarrow S$ and $t: N \rightarrow N$ be isometries. Then the direct sum $s \oplus t: S \oplus N \rightarrow$ $S \oplus N$ preserves the graph of $\kappa$ if and only if $\kappa \circ s=t \circ \kappa$.

Proposition 2.7 (see [13]). Let $S$ be a non-degenerate even lattice, let $s_{+}, s_{-}$be non-negative integers. Fix a subgroup $A \subset O(S)$. Then the A-isomorphism class of a primitive extension $L \supset S$ of $S$ to a unimodular lattice $L$ of signature $\left(s_{+}, s_{-}\right)$is determined by

(1) A choice of a lattice $N$ in the genus $\left(s_{+}-\sigma_{+} S, s_{-}-\sigma_{-} S ;-\mathcal{S}\right)$, and

(2) A choice of a bi-coset of the canonical left-right action of $A \times \operatorname{Aut}_{N} \mathcal{N}$ on the set of anti-isometries $\mathcal{S} \rightarrow \mathcal{N}$.

If a lattice $N$ and an anti-isometry $\kappa: \mathcal{S} \rightarrow \mathcal{N}$ as above are chosen (and thus an extension $L$ is fixed), an isometry $t: N \rightarrow N$ extends to an A-automorphism of $L$ if and only if the composition $\kappa^{-1} \circ t \circ \kappa \in$ Aut $\mathcal{S}$ is in the image of $A$.

Two primitive embeddings $\varphi_{1}, \varphi_{2}: S \rightarrow L$ are +-equivalent if there exists an isometry $t \in O^{*}(L)$ such that $t \circ \varphi_{1}=\varphi_{2}$.

Let $g(N)$ denote the set of isomorphism classes of non-degenerate integral lattice with same genus as $N$. The following statement is a corollary of Miranda, Morrison [11] and the previous statement.

Theorem 2.8. Let $S$ be a primitive non-degenerate sublattice of an even unimodular lattice $L$. Then there are exactly

$$
e(N)=\sum_{N \in g(N)}[\operatorname{Aut}(\mathcal{N}): \operatorname{Image}(O(N) \rightarrow \operatorname{Aut}(\mathcal{N}))]
$$

equivalence classes of primitive embeddings of $S$ into $L$ and exactly

$$
e^{*}(N)=\sum_{N \in g(N)}\left[\operatorname{Aut}(\mathcal{N}): \operatorname{Image}\left(O^{*}(N) \rightarrow \operatorname{Aut}(\mathcal{N})\right)\right]
$$

+-equivalence classes of such embeddings, where $N=S_{L}^{\perp}$ and $\mathcal{N}=\operatorname{disk}\left(S_{L}^{\perp}\right)$. 
A way to compute the numbers $e(N)$ and $e^{*}(N)$ is outlined in $[11,12]$. Note that, if $e(N)=1$ (respectively, $e^{*}(N)=1$ ), the canonical map $O(N) \rightarrow \operatorname{Aut}(\mathcal{N})$ (respectively, $\left.O^{*}(N) \rightarrow \operatorname{Aut}(\mathcal{N})\right)$ is onto.

\subsection{Moduli spaces}

A simple singularity can be defined as a singularity whose differential type is determined by its topological type. Isomorphism classes of simple singularities are known to be in a one to one correspondence (described below) with those of irreducible root systems (see Durfee [7] for details). Thus a set of simple singularities can be identified with a root system, namely the orthogonal direct sum of the irreducible summands. For this reason we use direct the summation symbol $\oplus$ in the notation.

Let $C \subset \mathbb{P}^{2}$ be a reduced sextic with simple singularities. Consider the double covering $X$ of $\mathbb{P}^{2}$ branched at $C$ and its minimal resolution of singularities $\bar{X} \rightarrow X$. It is known that $\bar{X}$ is a $K 3$ surface and the homology group $L_{X}=H_{2}(\bar{X} ; \mathbb{Z})$ together with the scalar product defined by the intersection index is isomorphic to $2 \mathbf{E}_{8} \oplus 3 \mathbf{U}$. The exceptional divisors in $\bar{X}$ contracted to a single singular point $p$ in $X$ are smooth rational $(-2)$-curves spanning in $L_{X}$ an irreducible root system corresponding to $p$. Hence the sublattice of $L_{X}$ spanned by all exceptional divisors is the set of singularities of $C$ (regarded as a root system as agreed above).

Introduce the following objects

$\sigma_{X} \subset L_{X}$ : the set of classes of exceptional divisors appeared in the blow-up $\bar{X} \rightarrow X$

$\Sigma_{X} \subset L_{X}$ : the sublattice generated by $\sigma_{X}$;

$h_{X} \in L_{X}$ : the pull-back of the class of a hyperplane section;

$S_{X}=\Sigma_{X} \oplus\left\langle h_{X}\right\rangle$ : the sublattice of $L_{X}$;

$\widetilde{\Sigma_{X}} \subset \widetilde{S}_{X} \subset L_{X}$ : the respective primitive hulls;

$\omega_{X} \subset \widetilde{S}_{X}^{\perp} \otimes \mathbb{R} \subset L_{X} \otimes \mathbb{R}$ : the oriented 2 -subspace spanned by the real and imaginary parts of a holomorphic 2 -form on $\bar{X}$. The orientation of $\omega_{X}$ is denoted by $\theta_{X}$.

An isomorphism between two triples $\left(L_{X_{i}}, h_{X_{i}}, \sigma_{X_{i}}\right), i=1,2$, as above is an isometry $L_{X_{1}} \rightarrow L_{X_{2}}$, taking $h_{X_{1}}$ to $h_{X_{2}}, \sigma_{X_{1}}$ to $\sigma_{X_{2}}$. The isomorphism class of the triple $\left(L_{X}, h_{X}, \sigma_{X}\right)$ associated to a sextic $C$ is called the homological type of $C$.

Definition 2.9. Let $\Sigma$ be a root system and $h$ a vector of square 2 . A configuration is a finite index extension $\widetilde{S} \supset S=\Sigma \oplus\langle h\rangle$ satisfying the following conditions:

(1) The primitive hull $\widetilde{\Sigma}=h \frac{\perp}{\widetilde{S}}$ of $\Sigma$ in $\widetilde{S}$ has no roots other than those in $\Sigma$;

(2) There is no root $r \in \Sigma$ such that $\frac{1}{2}(r+h) \in \widetilde{S}$.

Definition 2.10. For a fixed set of simple singularities $\Sigma$, an abstract homological type is an extension of $S=\Sigma \oplus\langle h\rangle,\left(h^{2}=2\right)$ to a lattice isomorphic to $2 \mathbf{E}_{8} \oplus 3 \mathbf{U}$, so that the primitive hull $\widetilde{S}$ of $S$ in $L$ is a configuration. 
Let $S=\Sigma \oplus\langle h\rangle, h^{2}=2$. One has $\mathcal{S}=\operatorname{disk} \Sigma \oplus\left\langle\frac{1}{2}\right\rangle$. An isometry of $S$ is called admissible if it preserves both $h$ and $\sigma$, and an isometry of $\widetilde{S}$ is called admissible if it preserves $S$ and induces an admissible isometry of $S$. Groups of admissible isometries of $S$ and $\widetilde{S}$ are denoted by $O_{h}(S)$ and $O_{h}(\widetilde{S})$, respectively. The image of the group of admissible isometries $O_{h}(\widetilde{S})$ in Aut $\widetilde{\mathcal{S}}$ is denoted by $\operatorname{Aut}_{h} \widetilde{\mathcal{S}}$. One has Aut $_{h} \widetilde{\mathcal{S}}=\left\{s \in \operatorname{Aut}_{h} \mathcal{S} \mid s(\mathcal{K})=\mathcal{K}\right\}$ where $\mathcal{K}$ is the kernel of the extension $\widetilde{S} \supset S$.

An orientation of an abstract homological type $\mathcal{H}=(L, h, \sigma)$ is one of two orientations of positive definite 2 -subspaces of $\widetilde{S}^{\perp} \otimes \mathbb{R}$. For a fixed orientation $\theta$, the abstract homological type $\mathcal{H}$ is called symmetric if $(\mathcal{H}, \theta)$ is isomorphic to $(\mathcal{H},-\theta)$. In other words, $\mathcal{H}$ is symmetric if it has an automorphism $t$ such that the restriction of $t$ to $\widetilde{S}^{\perp}$ is a + -disorienting isometry.

The following theorem is essential for classifying sextics with simple singularities.

Theorem 2.11 (see [4, Theorem 3.4.2]). The map sending a plane sextic $C \subset$ $\mathbb{P}^{2}$ to the pair consisting of its homological type $\left(L_{X}, h_{X}, \sigma_{X}\right)$ and the orientation $\theta_{X}$ establishes a one-to-one correspondence between the set of rigid isotopy classes of sextics with a given set of singularities $\Sigma$ and the set of isomorphism classes of oriented abstract homological types extending $\Sigma$.

\subsection{Zariski pairs}

Original definition of Zariski pair in [1] is as follows.

Definition 2.12. Two reduced curves $C_{1}, C_{2} \subset \mathbb{P}^{2}$ are said to form a Zariski pair if

- $\left(T_{1}, C_{1}\right)$ and $\left(T_{2}, C_{2}\right)$ are diffeomorphic where $T_{i}$ is a regular neighborhood of $C_{i}, i=1,2$, and

- the pairs $\left(\mathbb{P}^{2}, C_{1}\right)$ and $\left(\mathbb{P}^{2}, C_{2}\right)$ are not homeomorphic.

Definition 2.13. Two reduced curves $C_{1}, C_{2} \subset \mathbb{P}^{2}$ are said to have the same combinatorial data if there exist irreducible decompositions $C_{i}=C_{i, 1}+\cdots+C_{i, k_{i}}$, $i=1,2$, such that:

(1) $k_{1}=k_{2}$ and $\operatorname{deg} C_{1, j}=\operatorname{deg} C_{2, j}$ for all $j=1, \ldots, k_{1}$;

(2) There is a one-to-one correspondence between the singular points of $C_{1}$ and $C_{2}$, preserving the topological types of the points;

(3) Two singular points $P_{i} \in C_{i}, i=1,2$ corresponding to each other are related by a local homeomorphism such that if a branch at $P_{1}$ belongs to a component $B_{1, j}$ then its image belongs to $B_{2, j}$.

If the singularities of $C_{1}, C_{2}$ are simple then the first condition in Definition 2.12 can be replaced with the condition that the curves have the same combinatorial data. Another related definition is as follows. 
Definition 2.14. Two reduced curves $C_{1}, C_{2} \subset \mathbb{P}^{2}$ form a classical Zariski pair if

- $C_{1}$ and $C_{2}$ have the same combinatorial data, and

- the Alexander polynomials $\Delta_{C_{1}}(t), \Delta_{C_{2}}(t)$ differ.

Let $C_{1}, C_{2} \subset \mathbb{P}^{2}$ be a pair of irreducible sextics with the same set of simple singularities. As described in Sec. 1, Alexander polynomials of $C_{1}, C_{2}$ differ if and only if one is abundant and the other is not. The following statement is a crucial step in checking the existence of abundant and non-abundant types for a given set of singularities.

Theorem 2.15 (see [3, 4]). Each set of singularities $\Sigma$ as in (1.1) extends to two isomorphism classes of configurations $\widetilde{S} \supset S=\Sigma \oplus\langle h\rangle$ that may correspond to irreducible sextics, one abundant $(\mathcal{K}=\mathbb{Z} / 3 \mathbb{Z})$ and one not $(\mathcal{K}=0)$.

For any set of singularities, if there exist two abstract homological types, one with a configuration $\widetilde{S} \supset S$ such that $\widetilde{S} / S \cong \mathbb{Z} / 3 \mathbb{Z}$ and one with $\widetilde{S} / S \cong 0$, then this set of singularities is realized by two irreducible plane sextics which have the same combinatorial data and different Alexander polynomials. This is an efficient way to find classical Zariski pairs of sextics.

\subsection{Enumerating abstract homological types}

An approach to the classification of the oriented abstract homological types extending a given set of singularities $\Sigma$ is outlined in [4]. It can be carried out in four steps.

(1) Enumerating the configurations $\widetilde{S}$ extending $\Sigma$. Due to Proposition 2.6, a configuration is determined by a choice $\mathcal{K}=0$ or $\mathcal{K}=\mathbb{Z} / 3 \mathbb{Z}$.

(2) Enumerating the isomorphism classes of $\widetilde{S}^{\perp}$. Orthogonal complement of $\widetilde{S}$ has genus $(2,19-\operatorname{rk} \Sigma ;-\widetilde{\mathcal{S}})$. The existence of a lattice in this genus can be checked by Theorem 2.3 and its uniqueness can be checked by Theorems 2.4 and 2.5.

(3) Enumerating the bi-cosets of $\operatorname{Aut}_{h} \widetilde{S} \times \operatorname{Aut}_{N} \mathcal{N}$. Once the lattice $\widetilde{S}^{\perp}=N$ is chosen, one can fix an anti-isometry $\widetilde{\mathcal{S}} \rightarrow \mathcal{N}$ for $\mathcal{N}=\operatorname{disk} \widetilde{S}^{\perp}$ and, hence there exists an isomorphism Aut $\mathcal{N}=\operatorname{Aut} \widetilde{\mathcal{S}}$. Then the extensions are classified by the quotient set $\operatorname{Aut}_{h} \widetilde{\mathcal{S}} \backslash$ Aut $\widetilde{\mathcal{S}} / \operatorname{Aut}_{N} \mathcal{N}$.

(4) Detecting whether the abstract homological types are symmetric. An abstract homological type is symmetric if and only if $N$ has a +-disorienting isometry $t$ whose image in Aut $\mathcal{N}=$ Aut $\widetilde{\mathcal{S}}$ belongs to the product of the image of subgroup $O_{h}(\widetilde{S})$ and the image of $O^{*}(N)$.

The following statement provides a sufficient condition for an abstract homological type to be symmetric.

Proposition 2.16 (see [4]). Let $\mathcal{H}=(L, h, \sigma)$ be an abstract homological type. If the lattice $\widetilde{S}^{\perp}$ contains a vector $v$ of square 2 , then $\mathcal{H}$ is symmetric. 
On the other hand, instead of the above steps, by the following statement one can obtain unique abstract homological type for each configuration $\widetilde{S}$.

Theorem 2.17. Each configuration extending a set of singularities $\Sigma$ satisfying the inequality $\ell(\operatorname{disk} \Sigma)+\operatorname{rk} \Sigma \leq 19$ is realized by a unique rigid isotopy class of plane sextics.

\section{Proof of Theorem $\mathbf{1 . 1}$}

In this section we complete the classification of classical Zariski pairs. The case $n=0$ was settled in [4]. In the remaining part, there are 36 different sets of singularities obtainable from (1.1) with $n \neq 0$. By Theorem 2.15 each set of singularities should be examined in two types, abundant and non-abundant. Therefore, there are 72 different configurations to be investigated.

In Table 1, the last eight sets of singularities (with the bullet sign) satisfy the inequality $\ell(\operatorname{disk} \Sigma)+\operatorname{rk} \Sigma \leq 19$. They are definitely realized by two types of irreducible sextics (abundant and non-abundant) by Theorem 2.17. For the others we follow the steps listed in Subsec. 2.4.

The first step, the choice $\mathcal{K}=0$ or $\mathcal{K}=\mathbb{Z} / 3 \mathbb{Z}$ is straightforward according to Theorem 2.15. According to this choice, one has $\widetilde{\mathcal{S}}=\mathcal{S}$ or $|\widetilde{S}|=|S| / 9$, respectively.

In the second step, we look for the existence and uniqueness of a lattice $N$ to serve as the orthogonal complement of $\widetilde{S}$ in $L$. In Table 1, the cases for which the genus of $N$ (assuming the existence) does or does not satisfy the hypothesis of Theorems 2.3-2.5 are shown by the plus or minus signs, respectively. By Theorem 2.3 , the four sets

$$
6 \mathbf{A}_{2} \oplus 4 \mathbf{A}_{1}, \quad 2 \mathbf{A}_{2} \oplus 2 \mathbf{E}_{6} \oplus 2 \mathbf{A}_{1}, \quad 4 \mathbf{A}_{2} \oplus \mathbf{E}_{6} \oplus 3 \mathbf{A}_{1}, \quad 3 \mathbf{E}_{6} \oplus \mathbf{A}_{1}
$$

are realized by abundant curves only whereas any other set of singularities as in (1.1) is realized by both abundant and non-abundant curves. Hence only abundant types of these sets of singularities exist. For the uniqueness, it suffices to show that the lattice $N$ found satisfies the hypothesis of Theorem 2.4 or Theorem 2.5. As stated in Table 1 , this is the case for all sets of singularities except $\Sigma=3 \mathbf{E}_{6} \oplus \mathbf{A}_{1}$. The latter has a definite lattice $N$ of rank 2 in which case Theorems 2.4 and 2.5 are not applicable; however the existence of $N$ and uniqueness in its genus can easily be shown using Gauss [9].

The third step is to enumerate the bi-cosets of $\operatorname{Aut}_{h} \widetilde{\mathcal{S}} \times \operatorname{Aut}_{N} \mathcal{N}$. To have a unique class for each $S$, it is sufficient to have the homomorphism $O(N) \longrightarrow$ Aut $\mathcal{N}$ surjective, i.e. $\operatorname{Aut}_{N} \mathcal{N}=\operatorname{Aut} \mathcal{N}$. The set of singularities satisfying the hypothesis of Theorem 2.5 have this homomorphism surjective. They are listed in Table 1. Others have $e(N)=1$ (see Theorem 2.8) by the calculations in [11] and so their configurations also have this homomorphism surjective. Hence each abundant and non-abundant extensions of (1.1) other than (3.1) and each abundant extensions of (3.1) give rise to a unique abstract homological type. 
Table 1.

\begin{tabular}{|c|c|c|c|c|c|c|c|c|}
\hline \multirow[t]{2}{*}{ Singularities } & \multicolumn{4}{|c|}{ Abundant } & \multicolumn{4}{|c|}{ Non-abundant } \\
\hline & 2.3 & 2.4 & 2.5 & $\mathrm{e}\left(\widetilde{S}^{\perp}\right)$ & 2.3 & 2.4 & 2.5 & $\mathrm{e}\left(\widetilde{S}^{\perp}\right)$ \\
\hline$\overline{\Sigma_{1}=\mathbf{A}_{17} \oplus \mathbf{A}_{1}}$ & + & + & & & + & + & & \\
\hline$\Sigma_{3}=\mathbf{A}_{14} \oplus \mathbf{A}_{2} \oplus 2 \mathbf{A}_{1}$ & + & + & & & + & - & + & 1 \\
\hline$\Sigma_{4}=\mathbf{A}_{11} \oplus \mathbf{E}_{6} \oplus \mathbf{A}_{1}$ & + & - & + & 1 & + & - & + & 1 \\
\hline$\Sigma_{6}=\mathbf{A}_{11} \oplus 2 \mathbf{A}_{2} \oplus 2 \mathbf{A}_{1}$ & + & + & & & + & - & + & 1 \\
\hline$\Sigma_{7}=\mathbf{A}_{11} \oplus \mathbf{A}_{5} \oplus \mathbf{A}_{1}$ & + & + & & & + & + & & \\
\hline$\Sigma_{8}=\mathbf{A}_{8} \oplus \mathbf{E}_{6} \oplus \mathbf{A}_{2} \oplus \mathbf{A}_{1}$ & + & + & & & + & - & + & 1 \\
\hline$\Sigma_{9}=\mathbf{A}_{8} \oplus \mathbf{E}_{6} \oplus \mathbf{A}_{2} \oplus 2 \mathbf{A}_{1}$ & + & + & & & + & - & + & 1 \\
\hline$\Sigma_{11}=\mathbf{A}_{8} \oplus 3 \mathbf{A}_{2} \oplus 2 \mathbf{A}_{1}$ & + & + & & & + & - & + & 1 \\
\hline$\Sigma_{12}=\mathbf{A}_{8} \oplus 3 \mathbf{A}_{2} \oplus 3 \mathbf{A}_{1}$ & + & + & & & + & - & + & 1 \\
\hline$\Sigma_{14}=\mathbf{A}_{8} \oplus \mathbf{A}_{5} \oplus \mathbf{A}_{2} \oplus 2 \mathbf{A}_{1}$ & + & + & & & + & - & + & 1 \\
\hline$\Sigma_{16}=2 \mathbf{A}_{8} \oplus 2 \mathbf{A}_{1}$ & + & + & & & + & - & + & 1 \\
\hline$\Sigma_{17}=3 \mathbf{A}_{5} \oplus \mathbf{A}_{1}$ & + & + & & & + & + & & \\
\hline$\Sigma_{18}=2 \mathbf{A}_{5} \oplus \mathbf{E}_{6} \oplus \mathbf{A}_{1}$ & + & + & & & + & - & + & 1 \\
\hline$\Sigma_{20}=2 \mathbf{A}_{5} \oplus 2 \mathbf{A}_{2} \oplus 2 \mathbf{A}_{1}$ & + & + & & & + & - & + & 1 \\
\hline$\Sigma_{21}=\mathbf{A}_{5} \oplus 2 \mathbf{E}_{6} \oplus \mathbf{A}_{1}$ & + & + & & & + & - & + & 1 \\
\hline$\Sigma_{23}=\mathbf{A}_{5} \oplus 4 \mathbf{A}_{2} \oplus 2 \mathbf{A}_{1}$ & + & + & & & + & - & + & 1 \\
\hline$\Sigma_{24}=\mathbf{A}_{5} \oplus 4 \mathbf{A}_{2} \oplus 3 \mathbf{A}_{1}$ & + & + & & & + & - & + & 1 \\
\hline$\Sigma_{25}=\mathbf{A}_{5} \oplus \mathbf{E}_{6} \oplus 2 \mathbf{A}_{2} \oplus \mathbf{A}_{1}$ & + & + & & & + & - & + & 1 \\
\hline$\Sigma_{26}=\mathbf{A}_{5} \oplus \mathbf{E}_{6} \oplus 2 \mathbf{A}_{2} \oplus 2 \mathbf{A}_{1}$ & + & + & & & + & - & + & 1 \\
\hline$\Sigma_{28}=6 \mathbf{A}_{2} \oplus 2 \mathbf{A}_{1}$ & + & + & & & + & - & + & 1 \\
\hline$\Sigma_{29}=6 \mathbf{A}_{2} \oplus 3 \mathbf{A}_{1}$ & + & + & & & + & - & + & 1 \\
\hline$\Sigma_{30}=6 \mathbf{A}_{2} \oplus 4 \mathbf{A}_{1}$ & + & - & + & 1 & \multicolumn{4}{|c|}{ does not exist } \\
\hline$\Sigma_{31}=2 \mathbf{A}_{2} \oplus 2 \mathbf{E}_{6} \oplus \mathbf{A}_{1}$ & + & + & & & + & - & + & 1 \\
\hline$\Sigma_{32}=2 \mathbf{A}_{2} \oplus 2 \mathbf{E}_{6} \oplus 2 \mathbf{A}_{1}$ & + & - & + & 1 & \multicolumn{4}{|c|}{ does not exist } \\
\hline$\Sigma_{33}=4 \mathbf{A}_{2} \oplus \mathbf{E}_{6} \oplus \mathbf{A}_{1}$ & + & + & & & + & - & + & 1 \\
\hline$\Sigma_{34}=4 \mathbf{A}_{2} \oplus \mathbf{E}_{6} \oplus 2 \mathbf{A}_{1}$ & + & + & & & + & - & + & 1 \\
\hline$\Sigma_{35}=4 \mathbf{A}_{2} \oplus \mathbf{E}_{6} \oplus 3 \mathbf{A}_{1}$ & + & - & + & 1 & \multicolumn{4}{|c|}{ does not exist } \\
\hline$\Sigma_{36}=3 \mathbf{E}_{6} \oplus \mathbf{A}_{1}$ & + & \multicolumn{3}{|c|}{ not applicable } & \multicolumn{4}{|c|}{ does not exist } \\
\hline \multicolumn{9}{|l|}{$\bullet \Sigma_{2}=\mathbf{A}_{14} \oplus \mathbf{A}_{2} \oplus \mathbf{A}_{1}$} \\
\hline \multicolumn{9}{|l|}{ - $\Sigma_{5}=\mathbf{A}_{11} \oplus 2 \mathbf{A}_{2} \oplus \mathbf{A}_{1}$} \\
\hline \multicolumn{9}{|l|}{$\cdot \Sigma_{10}=\mathbf{A}_{8} \oplus 3 \mathbf{A}_{2} \oplus \mathbf{A}_{1}$} \\
\hline \multicolumn{9}{|l|}{ - $\Sigma_{13}=\mathbf{A}_{8} \oplus \mathbf{A}_{5} \oplus \mathbf{A}_{2} \oplus \mathbf{A}_{1}$} \\
\hline \multicolumn{9}{|l|}{ - $\Sigma_{15}=2 \mathbf{A}_{8} \oplus \mathbf{A}_{1}$} \\
\hline \multicolumn{9}{|l|}{$\cdot \Sigma_{19}=2 \mathbf{A}_{5} \oplus 2 \mathbf{A}_{2} \oplus \mathbf{A}_{1}$} \\
\hline \multicolumn{9}{|l|}{ - $\Sigma_{22}=\mathbf{A}_{5} \oplus 4 \mathbf{A}_{2} \oplus \mathbf{A}_{1}$} \\
\hline$\bullet \Sigma_{27}=6 \mathbf{A}_{2} \oplus \mathbf{A}_{1}$ & & & & & & & & \\
\hline
\end{tabular}

The last step is to check whether the abstract homological types are symmetric. The sets of singularities whose lattices $N$ contain a $\langle 2\rangle$-summand are realized by symmetric rigid isotopy type by Proposition 2.16. By the uniqueness of $N$ just proved, it suffices to examine a particular representative of each genus. All lattices can easily be written down and one can see that Proposition 2.16 applies to abundant cases in Table 2 and to the non-abundant case $\Sigma_{1}$. 
Table 2 .

\begin{tabular}{|c|c|c|c|}
\hline \multirow[t]{2}{*}{ Singularities } & \multicolumn{3}{|c|}{ Non-abundant } \\
\hline & Theorem 2.16 & $\mathrm{e}^{*}\left(\widetilde{S}^{\perp}\right)$ & \\
\hline$\overline{\Sigma_{1}=\mathbf{A}_{17} \oplus \mathbf{A}_{1}}$ & + & & \\
\hline$\Sigma_{3}=\mathbf{A}_{14} \oplus \mathbf{A}_{2} \oplus 2 \mathbf{A}_{1}$ & - & 2 & * \\
\hline$\Sigma_{4}=\mathbf{A}_{11} \oplus \mathbf{E}_{6} \oplus \mathbf{A}_{1}$ & - & 2 & * \\
\hline$\Sigma_{6}=\mathbf{A}_{11} \oplus 2 \mathbf{A}_{2} \oplus 2 \mathbf{A}_{1}$ & - & 1 & \\
\hline$\Sigma_{7}=\mathbf{A}_{11} \oplus \mathbf{A}_{5} \oplus \mathbf{A}_{1}$ & - & 1 & \\
\hline$\Sigma_{8}=\mathbf{A}_{8} \oplus \mathbf{E}_{6} \oplus \mathbf{A}_{2} \oplus \mathbf{A}_{1}$ & - & 1 & \\
\hline$\Sigma_{9}=\mathbf{A}_{8} \oplus \mathbf{E}_{6} \oplus \mathbf{A}_{2} \oplus 2 \mathbf{A}_{1}$ & - & 2 & $*$ \\
\hline$\Sigma_{11}=\mathbf{A}_{8} \oplus 3 \mathbf{A}_{2} \oplus 2 \mathbf{A}_{1}$ & - & 2 & * \\
\hline$\Sigma_{12}=\mathbf{A}_{8} \oplus 3 \mathbf{A}_{2} \oplus 3 \mathbf{A}_{1}$ & - & 2 & * \\
\hline$\Sigma_{14}=\mathbf{A}_{8} \oplus \mathbf{A}_{5} \oplus \mathbf{A}_{2} \oplus 2 \mathbf{A}_{1}$ & - & 1 & \\
\hline$\Sigma_{16}=2 \mathbf{A}_{8} \oplus 2 \mathbf{A}_{1}$ & - & 2 & * \\
\hline$\Sigma_{17}=3 \mathbf{A}_{5} \oplus \mathbf{A}_{1}$ & - & 1 & \\
\hline$\Sigma_{18}=2 \mathbf{A}_{5} \oplus \mathbf{E}_{6} \oplus \mathbf{A}_{1}$ & - & 1 & \\
\hline$\Sigma_{20}=2 \mathbf{A}_{5} \oplus 2 \mathbf{A}_{2} \oplus 2 \mathbf{A}_{1}$ & - & 2 & * \\
\hline$\Sigma_{21}=\mathbf{A}_{5} \oplus 2 \mathbf{E}_{6} \oplus \mathbf{A}_{1}$ & - & 2 & * \\
\hline$\Sigma_{23}=\mathbf{A}_{5} \oplus 4 \mathbf{A}_{2} \oplus 2 \mathbf{A}_{1}$ & - & 1 & \\
\hline$\Sigma_{24}=\mathbf{A}_{5} \oplus 4 \mathbf{A}_{2} \oplus 3 \mathbf{A}_{1}$ & - & 2 & $*$ \\
\hline$\Sigma_{25}=\mathbf{A}_{5} \oplus \mathbf{E}_{6} \oplus 2 \mathbf{A}_{2} \oplus \mathbf{A}_{1}$ & - & 2 & * \\
\hline$\Sigma_{26}=\mathbf{A}_{5} \oplus \mathbf{E}_{6} \oplus 2 \mathbf{A}_{2} \oplus 2 \mathbf{A}_{1}$ & - & 2 & * \\
\hline$\Sigma_{28}=6 \mathbf{A}_{2} \oplus 2 \mathbf{A}_{1}$ & - & 2 & * \\
\hline$\Sigma_{29}=6 \mathbf{A}_{2} \oplus 3 \mathbf{A}_{1}$ & - & 2 & * \\
\hline$\Sigma_{31}=2 \mathbf{A}_{2} \oplus 2 \mathbf{E}_{6} \oplus \mathbf{A}_{1}$ & - & 2 & * \\
\hline$\Sigma_{33}=4 \mathbf{A}_{2} \oplus \mathbf{E}_{6} \oplus \mathbf{A}_{1}$ & - & 1 & \\
\hline$\Sigma_{34}=4 \mathbf{A}_{2} \oplus \mathbf{E}_{6} \oplus 2 \mathbf{A}_{1}$ & - & 2 & $*$ \\
\hline
\end{tabular}

For the remaining sets of singularities, fix an anti-isometry $\widetilde{\mathcal{S}} \rightarrow \mathcal{N}$, identify Aut $\widetilde{\mathcal{S}} \cong$ Aut $\mathcal{N}$, and consider two homomorphisms

$$
O^{*}(N) \subseteq O(N) \stackrel{\varphi}{\longrightarrow} \operatorname{Aut}(\mathcal{N})=\operatorname{Aut}(\widetilde{\mathcal{S}}) \stackrel{\phi}{\longleftarrow} O(\widetilde{S}) \supseteq O_{h}(\widetilde{S})
$$

We are looking for a + -disorienting isometry $t \in O(N)$ such that image of $t$ in $\operatorname{Aut}(\mathcal{N})=\operatorname{Aut}(\widetilde{\mathcal{S}})$ belongs to the product of the image of subgroup $O_{h}(\widetilde{S})$ and the image of $O^{*}(N)$. Hence it suffices to have a +-disorienting isometry of $N$ which is also an element of the image of $O^{*}(N)$ or which is also an element of the image of $O_{h}(\widetilde{S})$. By the definition of $e^{*}(N)$ in Theorem 2.8, one can say that if $e^{*}(N)=1$, then $O^{*}(N) \longrightarrow \operatorname{Aut}(\mathcal{N})$ is surjective. For each set of singularities as in (1.1), there does exist a +-disorienting isometry of $N$ and so $\widetilde{\mathcal{S}}$ is symmetric whenever $e^{*}(N)=1$. In Table 2 , the cases which have $e^{*}(N) \neq 1$ are marked with “*” sign. In the following part, for each of these cases we look for a +-disorienting isometry whose image is an element in the image of $O_{h}(\widetilde{S})$.

Let $a$ be a direct summand of $N$ with $a^{2}>2$. Then the reflection $t_{a} \in O(N)$ is a + -disorienting isometry and it sends $a \in N$ to $-a$. We try to choose $a$ so that $\varphi\left(t_{a}\right)$ is the image of some automorphism in $O_{h}(\widetilde{S})$. In Table 3 , the discriminant groups $\mathcal{N}$ and the lattices $N$ of the sets of singularities marked with $*$ signs are listed, and 
Table 3 .

\begin{tabular}{lcc}
\hline Non-abundant $\Sigma$ & Disk $\widetilde{S}^{\perp}$ & $\widetilde{S}^{\perp}$ \\
\hline$\Sigma_{3}=\mathbf{A}_{14} \oplus \mathbf{A}_{2} \oplus 2 \mathbf{A}_{1}$ & $2\left\langle\frac{1}{2}\right\rangle \oplus\left\langle\frac{-1}{2}\right\rangle \oplus\left\langle\frac{2}{3}\right\rangle \oplus\left\langle\frac{-2}{3}\right\rangle \oplus\left\langle\frac{2}{5}\right\rangle$ & $\langle 6\rangle \oplus\langle-6\rangle \oplus\langle 10\rangle$ \\
$\Sigma_{4}=\mathbf{A}_{11} \oplus \mathbf{E}_{6} \oplus \mathbf{A}_{1}$ & $\left\langle\frac{1}{2}\right\rangle \oplus\left\langle\frac{-1}{2}\right\rangle \oplus\left\langle\frac{2}{3}\right\rangle \oplus\left\langle\frac{-2}{3}\right\rangle \oplus\left\langle\frac{1}{4}\right\rangle$ & $\langle 6\rangle \oplus\langle-6\rangle \oplus\langle 4\rangle$ \\
$\Sigma_{9}=\mathbf{A}_{8} \oplus \mathbf{E}_{6} \oplus \mathbf{A}_{2} \oplus 2 \mathbf{A}_{1}$ & $2\left\langle\frac{1}{2}\right\rangle \oplus\left\langle\frac{-1}{2}\right\rangle \oplus\left\langle\frac{2}{3}\right\rangle \oplus\left\langle\frac{-2}{3}\right\rangle \oplus\left\langle\frac{8}{9}\right\rangle$ & $\langle 6\rangle \oplus\langle-6\rangle \oplus\langle 18\rangle$ \\
$\Sigma_{11}=\mathbf{A}_{8} \oplus 3 \mathbf{A}_{2} \oplus 2 \mathbf{A}_{1}$ & $2\left\langle\frac{1}{2}\right\rangle \oplus\left\langle\frac{-1}{2}\right\rangle \oplus\left\langle\frac{2}{3}\right\rangle \oplus 2\left\langle\frac{-2}{3}\right\rangle \oplus\left\langle\frac{8}{9}\right\rangle$ & $\langle-6\rangle \oplus \mathbf{U}_{3} \oplus\langle-2\rangle \oplus\langle 18\rangle$ \\
$\Sigma_{12}=\mathbf{A}_{8} \oplus 3 \mathbf{A}_{2} \oplus 3 \mathbf{A}_{1}$ & $3\left\langle\frac{1}{2}\right\rangle \oplus\left\langle\frac{-1}{2}\right\rangle \oplus\left\langle\frac{2}{3}\right\rangle \oplus 2\left\langle\frac{-2}{3}\right\rangle \oplus\left\langle\frac{8}{9}\right\rangle$ & $\langle 6\rangle \oplus 2\langle-6\rangle \oplus\langle 18\rangle$ \\
$\Sigma_{16}=2 \mathbf{A}_{8} \oplus 2 \mathbf{A}_{1}$ & $2\left\langle\frac{1}{2}\right\rangle \oplus\left\langle\frac{-1}{2}\right\rangle \oplus 2\left\langle\frac{8}{9}\right\rangle$ & $\langle-2\rangle \oplus 2\langle 18\rangle$ \\
$\Sigma_{20}=2 \mathbf{A}_{5} \oplus 2 \mathbf{A}_{2} \oplus 2 \mathbf{A}_{1}$ & $2\left\langle\frac{1}{2}\right\rangle \oplus 3\left\langle\frac{-1}{2}\right\rangle \oplus 2\left\langle\frac{2}{3}\right\rangle \oplus 2\left\langle\frac{-2}{3}\right\rangle$ & $2\langle 6\rangle \oplus 2\langle-6\rangle \oplus\langle-2\rangle$ \\
$\Sigma_{21}=\mathbf{A}_{5} \oplus 2 \mathbf{E}_{6} \oplus \mathbf{A}_{1}$ & $\left\langle\frac{1}{2}\right\rangle \oplus 2\left\langle\frac{-1}{2}\right\rangle \oplus 2\left\langle\frac{2}{3}\right\rangle \oplus\left\langle\frac{-2}{3}\right\rangle$ & $2\langle 6\rangle \oplus\langle-6\rangle$ \\
$\Sigma_{24}=\mathbf{A}_{5}^{4} \oplus \mathbf{A}_{2} \oplus 3 \mathbf{A}_{1}$ & $3\left\langle\frac{1}{2}\right\rangle \oplus 2\left\langle\frac{-1}{2}\right\rangle \oplus 2\left\langle\frac{2}{3}\right\rangle \oplus 3\left\langle\frac{-2}{3}\right\rangle$ & $2\langle 6\rangle \oplus 3\langle-6\rangle$ \\
$\Sigma_{25}=\mathbf{A}_{5} \oplus \mathbf{E}_{6} \oplus 2 \mathbf{A}_{2} \oplus \mathbf{A}_{1}$ & $\left\langle\frac{1}{2}\right\rangle \oplus 2\left\langle\frac{-1}{2}\right\rangle \oplus 2\left\langle\frac{2}{3}\right\rangle \oplus 2\left\langle\frac{-2}{3}\right\rangle$ & $\langle 6\rangle \oplus\langle-6\rangle \oplus \mathbf{U}_{3} \oplus\langle-2\rangle$ \\
$\Sigma_{26}=\mathbf{A}_{5} \oplus \mathbf{E}_{6} \oplus 2 \mathbf{A}_{2} \oplus 2 \mathbf{A}_{1}$ & $2\left\langle\frac{1}{2}\right\rangle \oplus 2\left\langle\frac{-1}{2}\right\rangle \oplus 2\left\langle\frac{2}{3}\right\rangle \oplus 2\left\langle\frac{-2}{3}\right\rangle$ & $2\langle 6\rangle \oplus 2\langle-6\rangle$ \\
$\Sigma_{28}=6 \mathbf{A}_{2} \oplus 2 \mathbf{A}_{1}$ & $2\left\langle\frac{1}{2}\right\rangle \oplus\left\langle\frac{-1}{2}\right\rangle \oplus 2\left\langle\frac{2}{3}\right\rangle \oplus 4\left\langle\frac{-2}{3}\right\rangle$ & $2\langle-6\rangle \oplus 2 \mathbf{U}_{3} \oplus\langle-2\rangle$ \\
$\Sigma_{29}=6 \mathbf{A}_{2} \oplus 3 \mathbf{A}_{1}$ & $3\left\langle\frac{1}{2}\right\rangle \oplus\left\langle\frac{-1}{2}\right\rangle \oplus 2\left\langle\frac{2}{3}\right\rangle \oplus 4\left\langle\frac{-2}{3}\right\rangle$ & $\langle 6\rangle \oplus 3\langle-6\rangle \oplus \mathbf{U}_{3}$ \\
$\Sigma_{31}=2 \mathbf{A}_{2} \oplus 2 \mathbf{E}_{6} \oplus \mathbf{A}_{1}$ & $\left\langle\left\langle\frac{1}{2}\right\rangle \oplus\left\langle\frac{-1}{2}\right\rangle \oplus 2\left\langle\frac{2}{3}\right\rangle \oplus 2\left\langle\frac{-2}{3}\right\rangle\right.$ & $\langle 6\rangle \oplus\langle-6\rangle \oplus \mathbf{U}_{3}$ \\
$\Sigma_{34}=4 \mathbf{A}_{2} \oplus \mathbf{E}_{6} \oplus 2 \mathbf{A}_{1}$ & $2\left\langle\frac{1}{2}\right\rangle \oplus\left\langle\frac{-1}{2}\right\rangle \oplus 2\left\langle\frac{2}{3}\right\rangle \oplus 3\left\langle\frac{-2}{3}\right\rangle$ & $\langle 6\rangle \oplus 2\langle-6\rangle \oplus \mathbf{U}_{3}$ \\
\hline
\end{tabular}

we consider them one by one. Note that, due to the uniqueness of the embedding $\widetilde{S} \hookrightarrow L$, we can choose an anti-isometry $\widetilde{\mathcal{S}} \rightarrow \mathcal{N}$ arbitrarily, and in the discussion below we make the convenient choice after all necessary objects have been fixed.

- In $\Sigma_{3}, \Sigma_{20}, \Sigma_{24}, \Sigma_{25}, \Sigma_{26}, \Sigma_{29}, \Sigma_{31}, \Sigma_{34}$, let a denote the generator of a $\langle 6\rangle$ summand of $N$ and let $\alpha=\left[\frac{a}{3}\right], \beta=\left[\frac{a}{2}\right]$ denote the generators of $\left\langle\frac{1}{2}\right\rangle,\left\langle\frac{2}{3}\right\rangle$ summands of $\mathcal{N}$, respectively. One has the reflection $t_{a}: a \mapsto-a$ and $\varphi\left(t_{a}\right): \alpha \mapsto-\alpha$. By a proper choice of $\phi$, one can say that $\varphi\left(t_{a}\right)$ is induced under $\phi$ by a symmetry of the Dynkin graph of $\mathbf{A}_{2} \in \widetilde{S}$.

- In $\Sigma_{21}$, let $a_{1}$ and $a_{2}$ denote the generators of the two $\langle 6\rangle$ summands of $N$ and let $\alpha_{1}=\left[\frac{a_{1}}{3}\right], \alpha_{2}=\left[\frac{a_{2}}{3}\right]$ denote the generators of the two $\left\langle\frac{2}{3}\right\rangle$ summands. We can redecompose $\left\langle\alpha_{1}\right\rangle \oplus\left\langle\alpha_{2}\right\rangle \cong\left\langle\beta_{1}\right\rangle \oplus\left\langle\beta_{2}\right\rangle$, where $\beta_{1}=\alpha_{1}+\alpha_{2}$ and $\beta_{2}=\alpha_{1}-\alpha_{2}$ so that $\beta_{1}^{2}=\beta_{2}^{2}=-\frac{2}{3}$. One has the reflection $t_{a_{1}}: a_{1} \mapsto-a_{1}$ and its image $\varphi\left(t_{a_{1}}\right): \alpha_{1} \mapsto-\alpha_{1}$ acts via $\beta_{1} \mapsto-\beta_{2}, \beta_{2} \mapsto-\beta_{1}$. By a proper choice of $\phi$, one can say that $\varphi\left(t_{a_{1}}\right)$ is induced under $\phi$ by the transposition of the two $\mathbf{E}_{6}$ summands and a non-trivial symmetry of one of them.

- In $\Sigma_{28}$, let $u, v$ denote the standard basis of one of the $\mathbf{U}_{3}$ summand in $N$ and let $\alpha=\left[\frac{u+v}{3}\right], \beta=\left[\frac{u-v}{3}\right]$ denote the generators of the $\left\langle\frac{2}{3}\right\rangle,\left\langle\frac{-2}{3}\right\rangle$ summands in $\mathcal{N}$, respectively. Say $a=u+v$, then $t_{a}: a \mapsto-a$ is well-defined and $\varphi\left(t_{a}\right): \alpha \mapsto-\alpha$. By a proper choice of $\phi$, one can say that $\varphi\left(t_{a}\right)$ is induced under $\phi$ by a symmetry of the Dynkin graph of $\mathbf{A}_{2} \in \widetilde{S}$.

- In $\Sigma_{9}, \Sigma_{11}, \Sigma_{12}, \Sigma_{16}$, let $a$ denote the generator of the $\langle 18\rangle$ summand in $N$ and let $\alpha=\left[\frac{2 a}{9}\right], \beta=\left[\frac{a}{2}\right]$ denote the generators of $\left\langle\frac{8}{9}\right\rangle,\left\langle\frac{1}{2}\right\rangle$ summands in $\mathcal{N}$, respectively. One has $t_{a}: a \mapsto-a$ and $\varphi\left(t_{a}\right): \alpha \mapsto-\alpha$. By a proper choice of $\phi$, one can say that $\varphi\left(t_{a}\right)$ is induced under $\phi$ by a symmetry of the Dynkin graph of $\mathbf{A}_{8} \in \widetilde{S}$. 
Proposition 3.1. The set of singularities $\Sigma_{4}=\mathbf{A}_{11} \oplus \mathbf{E}_{6} \oplus \mathbf{A}_{1}$ extends to a nonabundant homological type which is asymmetric.

Proof. It is sufficient to describe the set of automorphisms $\phi\left(O_{h}(\widetilde{S})\right) \times \varphi\left(O^{*}(N)\right) \subseteq$ $\operatorname{Aut}(\mathcal{N})$, see $(3.2)$, and show that this set does not include the image of any +-disorienting isometry in $N$.

One has disk $\widetilde{S}^{\perp}=\mathcal{N}=\left\langle\frac{1}{2}\right\rangle \oplus\left\langle\frac{-1}{2}\right\rangle \oplus\left\langle\frac{2}{3}\right\rangle \oplus\left\langle\frac{-2}{3}\right\rangle \oplus\left\langle\frac{1}{4}\right\rangle$ and $\sigma_{N}=(2,1)$ and one can take $\widetilde{S}^{\perp}=N=\langle 6\rangle \oplus\langle-6\rangle \oplus\langle 4\rangle$. To find all possible isometries of $N$, one can make use of a larger lattice $N_{2}=\langle 2\rangle \oplus\langle-2\rangle \oplus\langle 4\rangle$ and its rescaling $\left(\frac{1}{2}\right) N_{2}$ denoted by $N_{1}=\langle 1\rangle \oplus\langle-1\rangle \oplus\langle 2\rangle \cong\langle 1\rangle \oplus\langle 1\rangle \oplus\langle-2\rangle$.

Let $a, b, c$ be the generators of the $\langle 6\rangle,\langle-6\rangle,\langle 4\rangle$-summands in $N$, respectively. Consider the generators $\delta=\left[\frac{b}{2}\right], \theta=\left[\frac{a}{2}\right], \alpha=\left[\frac{a}{3}\right], \beta=\left[\frac{b}{3}\right], \gamma=\left[\frac{c}{4}\right]$ of the $\left\langle\frac{1}{2}\right\rangle,\left\langle\frac{-1}{2}\right\rangle$, $\left\langle\frac{2}{3}\right\rangle,\left\langle\frac{-2}{3}\right\rangle,\left\langle\frac{1}{4}\right\rangle$-summands of $\mathcal{N}$, respectively. Let $y_{1}, y_{2}$ and $y_{3}$ be the generators of the $\langle 2\rangle,\langle-2\rangle$ and $\langle 4\rangle$ summands of $N_{2}$, respectively; they can be chosen as $y_{1}=\frac{b-2 a}{3}, y_{2}=\frac{a-2 b}{3}, y_{3}=c$. Also let $x_{1}=y_{2}+y_{3}, x_{2}=y_{1}, x_{3}=2 y_{2}+y_{3}$ be the generators of $\langle 1\rangle,\langle 1\rangle$ and $\langle-2\rangle$ summands in $N_{1}$, respectively.

Obviously, $N_{2}$ is a finite index extension of $N$, and $N$ can be identified with $N=\left\{v \mid v \cdot\left(y_{1}-y_{2}\right)=0 \bmod 3, v \in N_{2}\right\}$. Let $\mathcal{K}$ denote the kernel of this extension. Then $\mathcal{K}$ is the 3 -primary part; namely the subgroup generated by $\alpha+\beta$, since the discriminant of $N_{2}, \mathcal{N}_{2}=\left\langle\frac{1}{2}\right\rangle \oplus\left\langle\frac{-1}{2}\right\rangle \oplus\left\langle\frac{1}{4}\right\rangle$ is the same as that of $\mathcal{N}$ without 3 -primary part. Let us define $\operatorname{Aut}_{\mathcal{K}} \mathcal{N}=\left\{t_{\mathcal{N}} \in \operatorname{Aut}(\mathcal{N}): t_{\mathcal{N}}\right.$ preserves $\left.\mathcal{K}\right\}$ and $O_{\mathcal{K}}(N)=\left\{t_{N} \in O(N): \varphi\left(t_{N}\right)\right.$ preserves $\left.\mathcal{K}\right\}$ to make a connection between lattices $N$ and $N_{2}$.

By the above constructions one has $O(N) \supset O_{\mathcal{K}}(N) \subset O\left(N_{2}\right)$, where $O_{\mathcal{K}} N$ is generated by the automorphisms of $N_{2}$ preserving $\left(y_{1}-y_{2}\right) \bmod 3 N_{2}$, and $O(N)$ is generated by $O_{\mathcal{K}} N$ and $t_{b}$. Indeed, the preserved part $\alpha+\beta$ can be sent to $\alpha \pm \beta$. Since $t_{b}:(\alpha+\beta) \mapsto(\alpha-\beta)$, any automorphism in $O(N)$ non-preserving $\alpha+\beta$ can be obtained by the composition of $t_{b}$ and some $t_{N} \in O_{\mathcal{K}}(N)$.

It remains to calculate $O(N)$. Obviously $O\left(N_{2}\right)=O\left(N_{1}\right)$ and according to Vinberg [15], any automorphism on $N_{1}=\langle 1\rangle \oplus\langle 1\rangle \oplus\langle-2\rangle$ is generated by reflections $t_{x_{2}}, t_{x_{1}-x_{2}}, t_{x_{3}-2 x_{1}}$ which are $t_{y_{1}}, t_{-y_{1}+y_{2}+y_{3}}, t_{-y_{3}}$ in terms of $y_{1}, y_{2}, y_{3}$. Hence one can find $O_{\mathcal{K}} N=$ a automorphisms of $N_{2}$ preserving $\left.\left(y_{1}-y_{2}\right) \bmod 3 N_{2}\right\}$ by these reflections.

Since $\left(y_{1}-y_{2}\right)^{2}=0 \bmod 6$, in $O\left(N_{2}\right)$ the image of $\left(y_{1}-y_{2}\right) \bmod 3 N_{2}$ is a $(\bmod 3)$-isotropic element in $N_{2} / 3 N_{2}$, all such elements being $\left(y_{1} \pm y_{2}\right),\left(y_{1} \pm y_{3}\right)$ in $N_{2} \bmod 3 N_{2}$. The action of $O\left(N_{2}\right)$ on these elements is given by the following table:

\begin{tabular}{ccccc}
\hline & $y_{1}-y_{2}$ & $y_{1}+y_{2}$ & $y_{1}+y_{3}$ & $y_{1}-y_{3}$ \\
\hline$t_{y_{1}}$ & $y_{1}+y_{2}$ & $y_{1}-y_{2}$ & $y_{1}-y_{3}$ & $y_{1}+y_{3}$ \\
$t_{-y_{1}+y_{2}+y_{3}}$ & $y_{1}-y_{2}$ & $y_{1}+y_{3}$ & $y_{1}+y_{2}$ & $y_{1}-y_{3}$ \\
$t_{-y_{3}}$ & $y_{1}-y_{2}$ & $y_{1}+y_{2}$ & $y_{1}-y_{3}$ & $y_{1}+y_{3}$ \\
\hline
\end{tabular}


Denote $G=O\left(N_{2}\right)$ and let $G \supset H$ be the stabilizer of $\left(y_{1}-y_{2}\right) \bmod 3$. Then $O_{\mathcal{K}} N=H$ and $G / H \cong \operatorname{orbit}\left\{y_{1}-y_{2}\right\}$. As mentioned above, $G$ is generated by the reflections $t_{y_{1}}, t_{-y_{1}+y_{2}+y_{3}}, t_{-y_{3}}$.

Fix

$$
\left\{\begin{array}{l}
1 \\
t_{y_{1}}, \\
t_{-y_{1}+y_{2}+y_{3}} \circ t_{y_{1}}, \\
t_{y_{1}} \circ t_{-y_{1}+y_{2}+y_{3}} \circ t_{y_{1}}
\end{array}\right.
$$

as representatives of the cosets $\bmod H$. Then $H$ is generated by

$$
\begin{aligned}
& t_{-y_{1}+y_{2}+y_{3}}, \\
& t_{-y_{3}}, \\
& t_{y_{1}} \circ t_{-y_{1}+y_{2}+y_{3}} \circ t_{y_{1}} \circ t_{-y_{1}+y_{2}+y_{3}} \circ t_{y_{1}} \circ t_{-y_{1}+y_{2}+y_{3}} \circ t_{y_{1}}, \\
& t_{y_{1}} \circ t_{-y_{1}+y_{2}+y_{3}} \circ t_{-y_{3}} \circ t_{y_{1}} \circ t_{-y_{1}+y_{2}+y_{3}} \circ t_{y_{1}}
\end{aligned}
$$

by the Reidemeister-Schreier method. Then, in terms of the generators of $N$, $O_{\mathcal{K}} N=H$ is generated by $t_{c}, t_{a-b+c}, X, Y$ where

$$
\begin{aligned}
& X:=t_{b-2 a} \circ t_{a-b+c} \circ t_{b-2 a} \circ t_{a-b+c} \circ t_{b-2 a} \circ t_{a-b+c} \circ t_{b-2 a}, \\
& Y:=t_{b-2 a} \circ t_{a-b+c} \circ t_{c} \circ t_{b-2 a} \circ t_{a-b+c} \circ t_{b-2 a} .
\end{aligned}
$$

Computing the matrix representations, one can easily see that $t_{a-b+c}=X$. Hence, $O_{\mathcal{K}}(N)$ is generated by $t_{c}, t_{a-b+c}, Y$ and $O(N)$ is generated by $t_{b}, t_{c}, t_{a-b+c}, Y$. On the other hand, we have $O(N) / O^{*}(N)=\mathbb{Z}_{2}$ and choosing $1, t_{c}$ as the coset representatives we can apply the Reidemeister-Schreier method. Then we obtain that $O^{*}(N)$ is generated by $t_{b}, Y, t_{c} t_{a-b+c}, t_{a-b+c} t_{c}, t_{c} Y t_{c}$.

The group of automorphisms of $\mathcal{N}$ is isomorphic to $\left(\mathbb{Z}_{2}\right)^{4}$. Let us call its generators $g_{1}, g_{2}, g_{3}, g_{4}$ so that $g_{1}$ is the automorphism multiplying $\alpha$ by $(-1), g_{2}$ multiplying $\beta$ by $(-1), g_{3}$ multiplying $\gamma$ by $(-1)$ and $g_{4}$ is the only automorphism of the 2-primary part taking $\delta+\theta+\gamma$ to $\gamma$. The group of admissible isometries of $\widetilde{S}$ is generated by the non-trivial symmetry of the Dynkin graph of $\mathbf{A}_{11}$ and non-trivial symmetry of the Dynkin graph of $\mathbf{E}_{6}$. Hence, $\phi$ can be chosen so that $\phi\left(O_{h}(\widetilde{S})\right)$ in $\operatorname{Aut}(\mathcal{N})$ is generated by $g_{1}, g_{3}$ and $g_{2}$.

To have the homological type symmetric, we need to find an element in $\varphi\left(O(N) \backslash O^{*}(N)\right) \cap \phi\left(O_{h}(\widetilde{S})\right) \times \varphi\left(O^{*}(N)\right)$. According to the calculations above we have the following statements:

* $\operatorname{Aut}(\mathcal{N})=\operatorname{Aut}(\widetilde{\mathcal{S}})$ is generated by $g_{1}, g_{2}, g_{3}, g_{4}$,

* $\phi\left(O_{h}(\widetilde{S})\right)$ is generated by $g_{2}, g_{1} g_{3}$,

* $\varphi\left(O^{*}(N)\right)$ is generated by $g_{2}, g_{1} g_{3}, g_{4}$. 
It is seen that $\phi\left(O_{h}(\widetilde{S})\right) \subset \varphi\left(O^{*}(N)\right)$. On the other hand $t_{c} \in O(N) \backslash O^{*}(N)$ such that $\varphi\left(t_{c}\right)=g_{3} \bmod \varphi\left(O^{*}(N)\right)$. Hence $\varphi\left(O(N) \backslash O^{*}(N)\right) \cap \varphi\left(O^{*}(N)\right)=\emptyset$ and there is no +-disorienting isometry whose image is in $\varphi\left(O^{*}(N)\right)$.

\section{Acknowledgments}

I am grateful to Alexander Degtyarev who attracted my attention to this topic, encouraged and helped me in preparation of this paper. I am also grateful to Rick Miranda who patiently answered my questions concerning the details in [11] and $[12]$.

\section{References}

[1] E. Artal, Sur les couples de Zariski, J. Algebr. Geom. 3(2) (1994) 223-247.

[2] A. Degtyarev, Alexander polynomial of an algebraic hypersurface, preprint LOMI, R-11-86 (1986) (in Russian).

[3] A. Degtyarev, Alexander polynomial of a curve of degree six, J. Knot Theory Ramifications 3 (1994) 439-454.

[4] A. Degtyarev, On deformations of singular plane sextics, J. Algebr. Geom. 17(1) (2008) 101-135.

[5] A. Degtyarev, Oka's conjecture on irreducible plane sextics, J. London Math. Soc. $\mathbf{7 8}(2)$ (2008) 329-351.

[6] A. Degtyarev, Classical Zariski pairs, J. Singularities 2 (2010) 51-55.

[7] A. H. Durfee, The signature of smoothing of complex surface singularities, Ann. Math. 232 (1978) 85-98.

[8] C. Eyral and M. Oka, On the fundamental groups of the complements of plane singular sextics, J. Math. Soc. Japan 57(1) (2005) 37-54.

[9] C. F. Gauss, Disquisitiones Arithmeticae (Springer-Verlag, New York, 1986). Translated and with a preface by Arthur A. Clarke, Revised by W. C. Waterhouse, C. Greither and A. W. Grootendorst and with a preface by Waterhouse.

[10] A. Libgober, Alexander polynomial of plane algebraic curves and cyclic multiple planes, Duke Math. J. 49(4) (1982) 833-851.

[11] R. Miranda and D. R. Morrison, The number of embeddings of integral quadratic forms I, Proc. Japan Acad. Ser. A Math. Sci. 61(10) (1985) 317-320.

[12] R. Miranda and D. R. Morrison, The number of embeddings of integral quadratic forms II, Proc. Japan Acad. Ser. A Math. Sci. 62(1) (1986) 29-32.

[13] V. V. Nikulin, Integer quadratic forms and some of their geometrical applications, Izv. Akad. Nauk SSSR, Ser. Mat 43 (1979) 111-177 (in Russian), Math. USSR-Izv. 43 (1980) 103-167.

[14] M. Oka and D. T. Pho, Classification of sextics of torus type, Tokyo J. Math. 25(2) (2002) 399-433.

[15] E. B. Vinberg, On groups of unit elements of certain quadratic forms, Math. USSRSbornik 87 (1972) 17-35.

[16] O. Zariski, On the irregularity of cyclic multiple planes, Ann. Math. 32 (1931) $485-511$. 